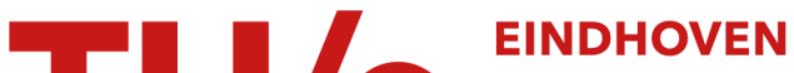 \\ UNIVERSITY OF \\ TECHNOLOGY
}

\section{Three lectures on metastability under stochastic dynamics}

Citation for published version (APA):

Hollander, den, W. T. F. (2007). Three lectures on metastability under stochastic dynamics. (Report Eurandom; Vol. 2007054). Eurandom.

Document status and date:

Published: 01/01/2007

\section{Document Version:}

Publisher's PDF, also known as Version of Record (includes final page, issue and volume numbers)

\section{Please check the document version of this publication:}

- A submitted manuscript is the version of the article upon submission and before peer-review. There can be important differences between the submitted version and the official published version of record. People interested in the research are advised to contact the author for the final version of the publication, or visit the $\mathrm{DOI}$ to the publisher's website.

- The final author version and the galley proof are versions of the publication after peer review.

- The final published version features the final layout of the paper including the volume, issue and page numbers.

Link to publication

\section{General rights}

Copyright and moral rights for the publications made accessible in the public portal are retained by the authors and/or other copyright owners and it is a condition of accessing publications that users recognise and abide by the legal requirements associated with these rights.

- Users may download and print one copy of any publication from the public portal for the purpose of private study or research.

- You may not further distribute the material or use it for any profit-making activity or commercial gain

- You may freely distribute the URL identifying the publication in the public portal.

If the publication is distributed under the terms of Article $25 \mathrm{fa}$ of the Dutch Copyright Act, indicated by the "Taverne" license above, please follow below link for the End User Agreement:

www.tue.nl/taverne

Take down policy

If you believe that this document breaches copyright please contact us at:

openaccess@tue.nl

providing details and we will investigate your claim. 


\title{
Three lectures on metastability under stochastic dynamics
}

\author{
Frank den Hollander ${ }^{12}$ \\ 1 Mathematical Institute \\ Leiden University \\ P.O. Box 9512 \\ 2300 RA Leiden \\ The Netherlands \\ denholla@math.leidenuniv.nl \\ 2 EURANDOM \\ P.O. Box 513 \\ 5600 MB Eindhoven \\ The Netherlands
}

Acknowledgment. The results described in these lectures are taken from joint work with Anton Bovier, Alex Gaudillière, Dima Ioffe, Francesca Nardi, Enzo Olivieri, Elisabetta Scoppola and Cristian Spitoni. Part of this work was supported by the Dutch-German Bilateral Research Group "Random Spatial Models from Physics and Biology", which is jointly funded by DFG and NWO. These lectures were also presented at the "School on Information and Randomness", Center for Mathematical Modeling, University of Chile at Santiago, 11-15 December 2006.

\section{Introduction}

Metastability is a phenomenon where a physical, chemical or biological system, under the influence of a noisy dynamics, moves between different regions of its state space on different time scales. On short time scales the system is in a quasi-equilibrium within a single region, while on long time scales it undergoes rapid transitions between quasi-equilibria in different regions (see Fig. 1).

Examples of metastability can be found in:

- biology: folding of proteins;

- climatology: effects of global warming;

- economics: crashes of financial markets;

- materials science: anomalous relaxation in disordered media;

- physics: freezing of supercooled liquids. 


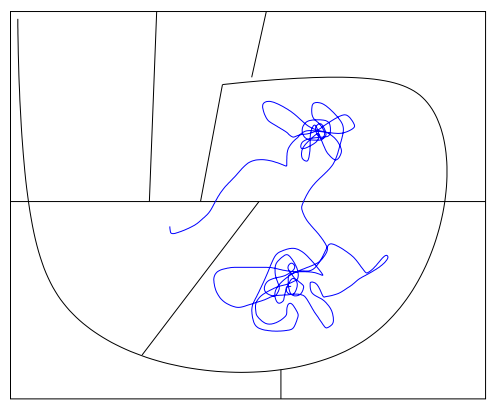

Fig. 1. The paradigm picture of metastability.

The task of mathematics is to formulate microscopic models of the relevant underlying dynamics, to prove the occurrence of metastable behavior in these models on macroscopic space-time scales, and to identify the key mechanisms behind the experimentally observed universality in the metastable behavior of whole classes of systems. This is a challenging program!

The mathematics of metastability started around 1935, with the work of Eyring, Kramers and Wigner on diffusions in potential wells. It further developed in the 1970's, through the work of Lebowitz and Penrose on metastable states in van der Waals theory [25] and Freidlin and Wentzell on randomly perturbed dynamical systems [15]. It accelerated in the 1980's with the implementation of Freidlin-Wentzell theory in statistical physics by Capocaccia, Cassandro, Galves, Kotecký, Martinelli, Neves, Olivieri, Schonmann, Scoppola and Vares. Presently, metastability is a highly active subfield of probability theory and statistical physics.

Two approaches to metastability are central within mathematics:

- Pathwise approach: This was initiated in 1984 by Cassandro, Galves, Olivieri and Vares [11], and is based on monitoring the full trajectory of the dynamics, in the spirit of Freidlin-Wentzell theory.

- Potential-theoretic approach: This was initiated in 2001 by Bovier, Eckhoff, Gayrard and Klein [5], [6], and is based on an electric network perspective of the dynamics, focussing on crossing times via estimates on capacities.

The latter approach is highlighted in the paper by Bovier in the present volume [4]. For recent overviews of metastability, see the monograph by Olivieri and Vares [24] and the review papers by den Hollander [17] and Bovier [3]. Earlier review papers include Penrose and Lebowitz [25], Schonmann [28], [29], Scoppola [31], Vares [32], Olivieri and Scoppola [23].

In Lectures 1-3 below we describe the metastable behavior of Ising spins subject to Glauber dynamics and of lattice gas particles subject to Kawasaki dynamics, both in two dimensions. Attention focusses on the identification of the geometry of the critical droplet for the crossover from the metastable state 
to the stable state, and on the estimation of the crossover time. We consider three cases:

(1) finite systems at low temperature;

(2) large systems at low temperature;

(3) moderate systems at positive temperature.

These cases are progressively more challenging, and for the latter two work is still in progress.

\section{Lecture 1: Finite systems at low temperature, definitions}

In Lecture 1, we define two models: (I) Ising spins subject to Glauber dynamics; (II) lattice gas particles subject to Kawasaki dynamics. We fix the metastable regimes of interest and introduce the notions of communication height and communication level set between metastable states. In Lecture 2, we formulate two theorems for these two models that quantify their metastable behavior.

\subsection{Glauber dynamics and Kawasaki dynamics}

Let $\Lambda \subset \mathbb{Z}^{2}$ be a large finite box. We consider two types of configurations:

(I) Ising spins: $\eta=\{\eta(x): x \in \Lambda\} \in \mathcal{X}=\{-1,+1\}^{\Lambda}$; $-1=$ down-spin, $+1=$ up-spin (see Fig. 2).

(II) Lattice gas: $\eta=\{\eta(x): x \in \Lambda\} \in \mathcal{X}=\{0,1\}^{\Lambda}$; $0=$ vacant, 1 = occupied (see Fig. 3 ).

$$
\begin{array}{|lllll|}
\hline- & - & + & - & - \\
+ & - & - & - & + \\
+ & - & - & + & - \\
- & + & + & + & - \\
+ & - & - & + & - \\
\hline
\end{array}
$$

Ising spins periodic boundary

Fig. 2. A configuration in model (I).

On the configuration space $\mathcal{X}$, we consider the following Hamiltonians assigning an energy to each configuration: 


\begin{tabular}{|lllll|}
\hline 0 & 0 & 1 & 0 & 0 \\
0 & 0 & 0 & 1 & 0 \\
0 & 1 & 1 & 0 & 0 \\
0 & 1 & 1 & 0 & 0 \\
0 & 0 & 0 & 0 & 0 \\
\hline
\end{tabular}

Lattice gas

open boundary

Fig. 3. A configuration in model (II).

$$
\begin{aligned}
& \text { (I) : } H(\eta)=-\frac{J}{2} \sum_{\substack{x, y \in \Lambda \\
x \sim y}} \eta(x) \eta(y)-\frac{h}{2} \sum_{x \in \Lambda} \eta(x), \\
& \text { (II) }: H(\eta)=-U \sum_{\substack{x, y \in \operatorname{int}(\Lambda) \\
x \sim y}} \eta(x) \eta(y)+\Delta \sum_{x \in \Lambda} \eta(x),
\end{aligned}
$$

where $\operatorname{int}(\Lambda)=\Lambda \backslash \partial \Lambda$ and $x \sim y$ means that $x$ and $y$ are neighboring sites. In (I) we pick periodic boundary conditions, in (II) we pick open boundary conditions (see (5)-(6) below). The parameters are:

(I) $J>0$ the ferromagnetic pair potential and $h \in \mathbb{R}$ the magnetic field;

(II) $U>0$ the binding energy and $\Delta>0$ the activation energy.

Definition 1.1. The Metropolis dynamics at inverse temperature $\beta \in(0, \infty)$ is the continuous-time Markov process $X=(X(t))_{t \geq 0}$ on $\mathcal{X}$ with transition rates

$$
c\left(\eta, \eta^{\prime}\right)=\exp \left\{-\beta\left[H\left(\eta^{\prime}\right)-H(\eta)\right]_{+}\right\}, \quad \eta, \eta^{\prime} \in \mathcal{X},
$$

(where $[\cdot]_{+}$denotes the positive part) and allowed transitions

$$
\begin{aligned}
& \text { (I) }: \eta^{\prime}=\eta^{x}, \quad x \in \Lambda, \\
& \text { (II) }: \eta^{\prime}=\eta^{x, y}, x, y \in \Lambda, x \sim y
\end{aligned}
$$

where

$$
\eta^{x}(y)=\left\{\begin{array}{l}
\eta(y), \quad y \neq x, \\
-\eta(x), y=x,
\end{array} \quad \eta^{x, y}(z)=\left\{\begin{array}{l}
\eta(z), z \neq x, y \\
\eta(x), z=y \\
\eta(y), z=x
\end{array}\right.\right.
$$

In words, for Ising spins the dynamics consists of spin-flips at single sites, called Glauber dynamics, while for the lattice gas it consists of exchange of occupation numbers between neighboring sites, called Kawasaki dynamics.

In the second dynamics, we also allow particles to enter and exit at $\partial \Lambda$. To that end, we also allow transitions

$$
\text { (II) : } \quad \eta^{\prime}=\eta^{*, x}, \quad x \in \partial \Lambda,
$$

where 


$$
\eta^{*, x}(y)= \begin{cases}\eta(y), & y \neq x \\ 1-\eta(x), & y=x\end{cases}
$$

View this as mimicking the presence of an infinite gas reservoir in $\mathbb{Z}^{2} \backslash \Lambda$ with density $e^{-\beta \Delta}$, which inserts particles at the sites of $\partial \Lambda$ at rate $e^{-\beta \Delta}$ and removes particles from the sites of $\partial \Lambda$ at rate 1 .

A key observation is the following. The Metropolis dynamics has the Gibbs measure

$$
\mu(\eta)=\frac{1}{Z} e^{-\beta H(\eta)}, \quad \eta \in \mathcal{X},
$$

with $Z$ the normalizing partition sum, as its reversible equilibrium, i.e.,

$$
\mu(\eta) c\left(\eta, \eta^{\prime}\right)=\mu\left(\eta^{\prime}\right) c\left(\eta^{\prime}, \eta\right), \quad \forall \eta, \eta^{\prime} \in \mathcal{X} .
$$

Note that the two Hamiltonians in (1) can be transformed into each other via the transformation

$$
\eta(x) \leftrightarrow \frac{1}{2}[1+\eta(x)], \quad h \leftrightarrow 2 U-\Delta, \quad J \leftrightarrow \frac{1}{2} U
$$

(modulo constant terms and boundary terms). However, the allowed transitions for the two dynamics cannot be transformed into each other. Indeed, the first dynamics is non-conservative, the second dynamics is conservative (except at the boundary $\partial \Lambda$ ).

In what follows, we write $\mathbb{P}_{\eta}$ to denote the law of $X$ given $X(0)=\eta$. For $\mathcal{A} \subset \mathcal{X}$, we write

$$
\tau_{\mathcal{A}}=\inf \{t \geq 0: X(t) \in \mathcal{A}, X(t-) \notin \mathcal{A}\}
$$

to denote the first entrance time of $\mathcal{A}$ by $X$.

\subsection{Metastable regimes}

We will study the two dynamics in the low temperature limit $\beta \rightarrow \infty$, in their so-called metastable regimes:

$$
\text { (I) }: 0<h<2 J, \quad \text { (II) }: U<\Delta<2 U .
$$

The dynamics will start in the configurations

$$
\text { (I) : } \boxminus=\text { all spins down, } \quad \text { (II) : } \square=\text { all sites vacant, }
$$

and we will be interested in how the dynamics tunnels to the configurations

$$
\text { (I) : } \boxplus=\text { all spins up, } \quad(\mathrm{II}): \mathbf{\square}=\text { all sites occupied. }
$$

To understand the restrictions in (11), let us consider the energy of an $\ell \times \ell$ droplet inside $\Lambda$, i.e., 


$$
(\mathrm{I}): E(\ell)=H\left(\eta_{\ell \times \ell}\right)-H(\boxminus), \quad(\mathrm{II}): E(\ell)=H\left(\eta_{\ell \times \ell}\right)-H(\square) .
$$

(Note that $H(\square)=0$, while $H(\boxminus)<0$ when $\Lambda$ is large enough depending on $J$ and $h$.) An easy computation gives

$$
\text { (I): } E(\ell)=J[4 \ell]-h \ell^{2}, \quad(\mathrm{II}): E(\ell)=-U[2 \ell(\ell-1)]+\Delta \ell^{2} .
$$

In both cases, $\ell \mapsto E(\ell)$ is a downward parabola that goes through a maximum at $\ell=\frac{2 J}{h}$, respectively, $\ell=\frac{U}{2 U-\Delta}$. Hence, if both these ratios are non-integer, then the critical droplets (i.e., the droplets with maximal energy on the parabola) are somewhere between a square of size $\ell_{c}-1$ and a square of size $\ell_{c}$, where

$$
(\mathrm{I}): \ell_{c}=\left\lceil\frac{2 J}{h}\right\rceil, \quad(\mathrm{II}): \ell_{c}=\left\lceil\frac{U}{2 U-\Delta}\right\rceil,
$$

are the critical droplet sizes. The regimes in (11) correspond to $\ell_{c} \in(1, \infty)$.

In configuration space, we have the following qualitative picture:

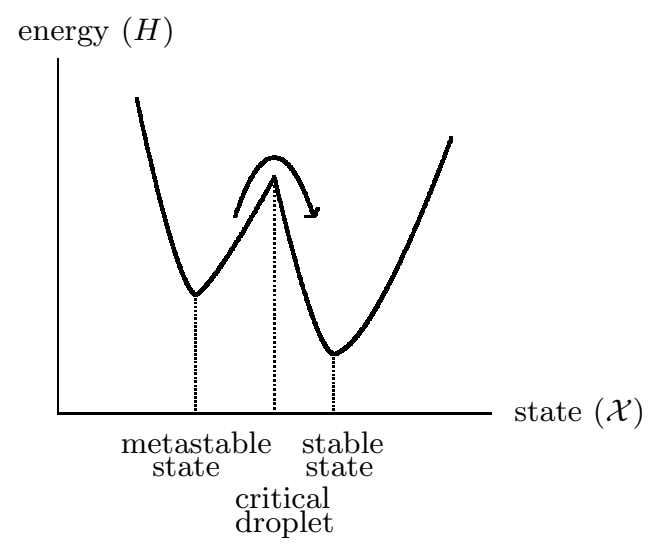

Fig. 4. The paradigm picture of the energy landscape.

The metastable regimes in (11) correspond to the situation where $\boxminus$ and $\square$ are local minima of the energy (lying at the bottom of a larger valley), $\boxplus$ and $\boldsymbol{\square}$ are global minima, and for the dynamics to move from one to the other it has to "go over an energetic hill". In physics language, we say that $\boxminus$ and $\square$ are metastable states, $\boxplus$ and $\boldsymbol{\square}$ are stable states, and the top of the hill separating them are critical droplets. We will address the following two questions (see Fig. 4):

(A) What are the critical droplets for the transitions $\boxminus \rightarrow \boxplus$ and $\square \rightarrow \mathbf{\square}$ ?

(B) How large are the crossover times $\tau_{\boxplus}$ and $\tau_{\square}$ starting from $\boxminus$ and $\square$, respectively? 


\subsection{Communication height and level set}

Write $\omega: \eta \rightarrow \eta^{\prime}$ to denote a path of allowed transitions from $\eta$ to $\eta^{\prime}$.

Definition 1.2. The communication height between $\boxminus$ and $\boxplus$ is defined as

$$
\text { (I) : } \Gamma=\Gamma(\boxminus, \boxplus)=\min _{\omega: \boxminus \rightarrow \boxplus} \max _{\xi \in \omega}[H(\xi)-H(\boxminus)] .
$$

The corresponding communication level set is

$$
\begin{aligned}
(\mathrm{I}): \mathcal{S}=\mathcal{S}(\boxminus, \boxplus)=\{\zeta \in \mathcal{X}: \exists \omega: \boxminus \rightarrow \boxplus \text { with } \omega \ni \zeta \text { such that } \\
\left.\max _{\xi \in \omega}[H(\xi)-H(\boxminus)]=H(\zeta)-H(\boxminus)=\Gamma\right\} .
\end{aligned}
$$

Similar definitions apply for $\square$, $\mathbf{\square}$ (with $H(\square)=0$ ).

In words, $\Gamma$ is the minimal amount the energy has to increase in a path that achieves the crossover, called the activation energy, while $\mathcal{S}$ is the set of all saddle point configurations in the path (recall Fig. 4).

Our intuitive guess for the answer to question (A) is that the critical droplets are the configurations in $\mathcal{S}$, and for the answer to question (B) that

$$
\tau_{\boxplus}, \tau_{\square} \approx e^{\beta \Gamma} \quad \text { as } \beta \rightarrow \infty .
$$

We will show in Lecture 2 that (19) is correct, obtaining in fact sharp estimates on $\mathbb{E}_{\boxminus}\left(\tau_{\boxplus}\right)$ and $\mathbb{E}_{\square}\left(\tau_{\mathbf{m}}\right)$, but that the critical droplets actually form a smaller set of configurations than $\mathcal{S}$, with an interesting geometry. We will see that models (I) and (II) show interesting similarities and differences.

\section{Lecture 2: Finite systems at low temperature, theorems}

In this lecture, we formulate two theorems that quantify the metastable behavior of models (I) and (II) in the regimes (11) by providing detailed answers to questions (A) and (B).

\subsection{Glauber dynamics}

Theorem 2.1. (Neves and Schonmann [22], Bovier and Manzo [10])

(a) There exists a set of configurations $\mathcal{C}^{*} \subsetneq \mathcal{S}$ such that

$$
\lim _{\beta \rightarrow \infty} \mathbb{P}_{\boxminus}\left(\tau_{\mathcal{C}^{*}}<\tau_{\boxplus} \mid \tau_{\boxplus}<\tau_{\boxminus}\right)=1,
$$

while this fails for any smaller set.

(b) The configurations in $\mathcal{C}^{*}$ are those where the up-spins form an $\ell_{c} \times\left(\ell_{c}-1\right)$ 
quasi-square, with a single protuberance on one of the sides of length $\ell_{c}$.

(c) The entrance distribution on $\mathcal{C}^{*}$ is asymptotically uniform:

$$
\lim _{\beta \rightarrow \infty} \mathbb{P}_{\boxminus}\left(X\left(\tau_{\mathcal{C}^{*}}\right)=\eta \mid \tau_{\mathcal{C}^{*}}<\tau_{\boxminus}\right)=\left|\mathcal{C}^{*}\right|^{-1} \quad \forall \eta \in \mathcal{C}^{*} .
$$

(d) There exists a constant $0<K=K\left(\Lambda, \ell_{c}\right)<\infty$ such that

$$
\lim _{\beta \rightarrow \infty} e^{-\beta \Gamma} \mathbb{E}_{\boxminus}\left(\tau_{\boxplus}\right)=K
$$

with

$$
\Gamma=H\left(\mathcal{C}^{*}\right)=J\left[4 \ell_{c}\right]-h\left[\ell_{c}\left(\ell_{c}-1\right)+1\right],
$$

and

$$
\lim _{\beta \rightarrow \infty} \mathbb{P}_{\boxminus}\left(\tau_{\boxplus}>t \mathbb{E}_{\boxminus}\left(\tau_{\boxplus}\right)\right)=e^{-t} \quad \forall t \geq 0 .
$$

(e) For all $\Lambda$,

$$
K\left(\Lambda, \ell_{c}\right)=\frac{3}{4\left(2 \ell_{c}-1\right)} \frac{1}{|\Lambda|} .
$$

Parts (a)-(b), together with the crude estimate $\lim _{\beta \rightarrow \infty}(1 / \beta) \log \mathbb{E}_{\boxminus}\left(\tau_{\boxplus}\right)=\Gamma$, were proved in [22]. Parts (c)-(e) were proved in [10].

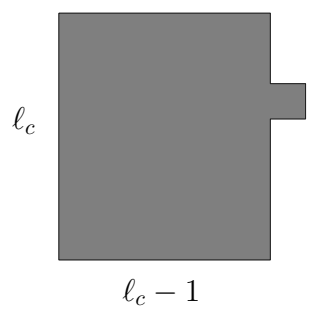

Fig. 5. A critical droplet for model (I): The up-spins lie inside the shaded area, the down-spins outside.

Theorem 2.1(a) says that the configurations in $\mathcal{C}^{*}$ are the critical droplets that represent the gate for the crossover. According to Theorem 2.1(c), the entrance distribution of this gate is uniform.

Theorem 2.1(b) is explained as follows (see Fig. 6). Since the dynamics flips one spin at a time, on its way from $\boxminus$ to $\boxplus$ it must pass through a configuration that has $\ell_{c}\left(\ell_{c}-1\right)$ up-spins. Among the configurations with precisely this number of up-spins, those where the up-spins form an $\ell_{c} \times\left(\ell_{c}-1\right)$ quasi-square (of any location and orientation) have the smallest energy (due to a discrete isoperimetric inequality; see e.g. Alonso and Cerf [1]). Continuing on its way from $\boxminus$ to $\boxplus$, the dynamics must flip one more spin upwards. The configurations with smallest energy are those where this spin is attached to one of the sides of the quasi-square, forming a protuberance (see Fig. 5). Next, 
if this protuberance sits on one of the sides of length $\ell_{c}$, then the dynamics can proceed downwards in energy by successively flipping up the spins next to the protuberance, to end up in an $\ell_{c} \times \ell_{c}$ square. This square is "over the hill" (see Fig. 4), because both its side lengths are supercritical (recall (16)). On the other hand, if the protuberance sits on one of the sides of length $\ell_{c}-1$, then the dynamics can proceed downwards in energy to form an $\left(\ell_{c}-1\right) \times\left(\ell_{c}+1\right)$ rectangle, but this rectangle is "not over the hill", because one of its side lengths is subcritical.

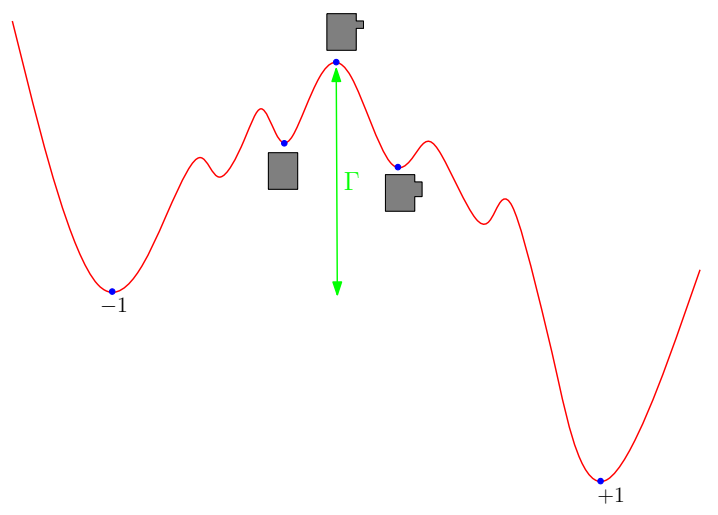

Fig. 6. A nucleation path.

Here is an explanation of Theorem 2.1(d-e). The exponential law comes from the fact that the crossover only occurs after many unsuccessful attempts to create a critical droplet and "go over the hill". The average time needed to enter $\mathcal{C}^{*}$ is

$$
\frac{1}{\left|\mathcal{C}^{*}\right|} e^{\beta \Gamma}[1+o(1)] \quad \text { as } \beta \rightarrow \infty .
$$

Let $\pi\left(\ell_{c}\right)$ denote the average probability with respect to the uniform entrance distribution that the critical droplet is exited in the direction of $\boxplus$ rather than $\boxminus$. Then the average number of attempts to go over the hill after reaching the top is

$$
\frac{1}{\pi\left(\ell_{c}\right)}[1+o(1)] \quad \text { as } \beta \rightarrow \infty .
$$

The product of (26) and (27) is the average crossover time, and so

$$
K=\frac{1}{\left|\mathcal{C}^{*}\right| \pi\left(\ell_{c}\right)} \text {. }
$$

Now,

$$
\left|\mathcal{C}^{*}\right|=|\Lambda| 4 \ell_{c}
$$

because the droplet can be centered anywhere in $\Lambda$, has 2 possible orientations, and the protuberance can sit in $2 \ell_{c}$ places. Moreover, 


$$
\pi\left(\ell_{c}\right)=\frac{1}{\ell_{c}}\left(2 \frac{1}{2}+\left(\ell_{c}-2\right) \frac{2}{3}\right) .
$$

Indeed, if the protuberance sits at one of the two extreme ends of a side of length $\ell_{c}$, then the probability is $\frac{1}{2}$ that its one neighboring spin on the same side flips upwards before the protuberance flips downwards. On the other hand, when the protuberance sits at one of the $\ell_{c}-2$ other locations on this side, then it has two neighboring spins on the same side and so the probability for one of them to flip upwards before the protuberance flips downwards is $\frac{2}{3}$. Combining (28-30), we get (25).

In Theorem 2.1(a), an example of a configuration in $\mathcal{S} \backslash \mathcal{C}^{*}$ is obtained by picking any configuration in $\mathcal{C}^{*}$, flipping down any spin next to the protuberance (at gain $h$ ) and afterwards flipping up any spin at a corner of the quasi-square (at cost $h$ ). For the dynamics, this configuration is a dead-end. Indeed, the last flip must be reversed before the dynamics can initiate the motion downhill to the $\ell_{c} \times \ell_{c}$ square.

\subsection{Kawasaki dynamics}

Theorem 2.2. (den Hollander, Olivieri and Scoppola [19], Bovier, den Hollander and Nardi [8])

(a) There exists a set of configurations $\mathcal{C}^{*} \subsetneq \mathcal{S}$ such that

$$
\lim _{\beta \rightarrow \infty} \mathbb{P}_{\square}\left(\tau_{\mathcal{C}^{*}}<\tau_{\mathbf{\square}} \mid \tau_{\mathbf{\square}}<\tau_{\square}\right)=1,
$$

while this fails for any smaller set.

(b) The configurations in $\mathcal{C}^{*}$ are those where the particles either form an $\left(\ell_{c}-2\right) \times\left(\ell_{c}-2\right)$ square, with four bars attached to the four sides of total length $3 \ell_{c}-3$ and 1 free particle, or form an $\left(\ell_{c}-1\right) \times\left(\ell_{c}-3\right)$ rectangle, with four bars attached to the four sides of total length $3 \ell_{c}-2$ and 1 free particle.

(c) The entrance distribution on $\mathcal{C}^{*}$ is asymptotically uniform:

$$
\lim _{\beta \rightarrow \infty} \mathbb{P}_{\square}\left(X\left(\tau_{\mathcal{C}^{*}}\right)=\eta \mid \tau_{\mathcal{C}^{*}}<\tau_{\square}\right)=\left|\mathcal{C}^{*}\right|^{-1} \quad \forall \eta \in \mathcal{C}^{*} .
$$

(d) There exists a constant $0<K=K\left(\Lambda, \ell_{c}\right)<\infty$ such that

$$
\lim _{\beta \rightarrow \infty} e^{-\beta \Gamma} \mathbb{E}_{\square}\left(\tau_{\mathbf{\square}}\right)=K
$$

with

$$
\Gamma=H\left(\mathcal{C}^{*}\right)=-U\left(2 \ell_{c}^{2}-4 \ell_{c}+2\right)+\Delta\left(\ell_{c}^{2}-\ell_{c}+2\right),
$$

and

$$
\lim _{\beta \rightarrow \infty} \mathbb{P}_{\square}\left(\tau_{\mathbf{\square}}>t \mathbb{E}_{\square}\left(\tau_{\mathbf{\square}}\right)\right)=e^{-t} \quad \forall t \geq 0 .
$$

(e) $A s \Lambda \rightarrow \mathbb{Z}^{2}$,

$$
K\left(\Lambda, \ell_{c}\right) \sim \frac{3}{4 \pi \ell_{c}^{2}\left(\ell_{c}^{2}-1\right)} \frac{\log |\Lambda|}{|\Lambda|} .
$$


Part (a), together with a partial description of $\mathcal{C}^{*}$ and the crude estimate $\lim _{\beta \rightarrow \infty}(1 / \beta) \log \mathbb{E}_{\boxminus}\left(\tau_{\boxplus}\right)=\Gamma$, were proved in [19]. Parts (b)-(e) were proved in $[8]$.

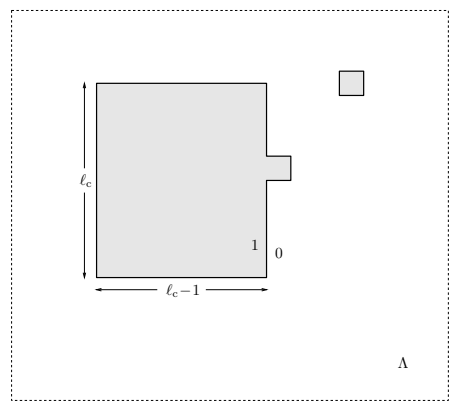

Fig. 7. A critical droplet for model (II): The occupied sites lie inside the shaded areas, the vacant sites outside.

Comparing Theorem 2.2(b) with Theorem 2.1(b), we see that the critical droplet for Kawasaki is more complicated than for Glauber. Once the dynamics has created a protocritical droplet (= quasi-square plus protuberance without free particle), it must wait for the next particle to arrive from the boundary (which is the free particle in Fig. 7). This takes a time of order $e^{\beta \Delta}$. Because $\Delta>U$, this time is much larger than $e^{\beta U}$, the time for the dynamics to make moves that cost $U$. Therefore the droplet will "explore" all shapes that can be reached from its protocritical shape via a $U$-path, i.e., a path between two configurations with the same energy that never goes more than $U$ above this energy. For instance, the protuberance may detach itself from the side of length $\ell_{c}$ and reattach itself to the side of length $\ell_{c}-1$. But it is also possible for particles to slide along the boundary of the droplet, in a train-like motion around corners (see Fig. 8), so as to modify the four bars in the annulus of the droplet.

Theorem $2.1(\mathrm{~d}-\mathrm{e})$ is explained as follows. Write $\mathcal{C}$ to denote the set of protocritcal droplets. The average time needed to enter $\mathcal{C}^{*}$ is

$$
\frac{1}{|\mathcal{C}||\partial \Lambda|} e^{\beta \Gamma}[1+o(1)] \quad \text { as } \beta \rightarrow \infty
$$

Let $\pi\left(\Lambda, \ell_{c}\right)$ denote the average probability with respect to the uniform entrance distribution that the critical droplet is exited in the direction of rather than $\square$. Then the average number of attempts to go over the hill after reaching the top is

$$
\frac{1}{\pi\left(\Lambda, \ell_{c}\right)}[1+o(1)] \quad \text { as } \beta \rightarrow \infty .
$$

The product of (26) and (27) is the average crossover time, and so 


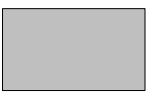

(1)

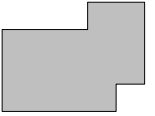

(6)

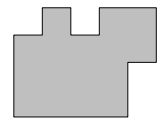

(11)

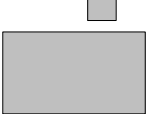

(2)

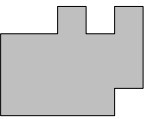

(7)

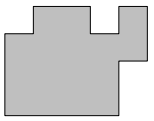

(12)

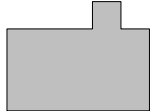

(3)

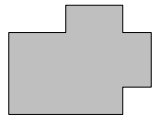

(8)

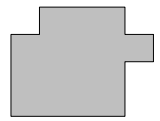

(13)

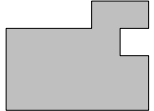

(4)

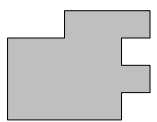

(9)

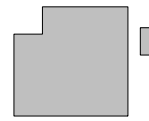

(14)

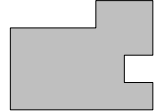

(5)

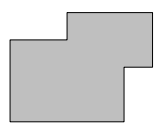

(10)

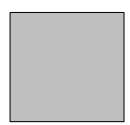

(15)

Fig. 8. Motion along the border of the droplet. Configurations (3-13) form a $U$-path.

$$
K=\frac{1}{|\mathcal{C}||\partial \Lambda| \pi\left(\Lambda, \ell_{c}\right)}
$$

Now,

$$
|\mathcal{C}| \sim|\Lambda| \frac{1}{3} \ell_{c}^{2}\left(\ell_{c}^{2}-1\right) \quad \text { as } \Lambda \rightarrow \mathbb{Z}^{2}
$$

where the first factor comes from centering the droplet anywhere in $\Lambda$ not touching $\partial \Lambda$, while the second factor comes from a combinatorial calculation counting the number of sizes and locations of the four bars in the annulus. Moreover,

$$
|\partial \Lambda| \pi\left(\ell_{c}, \Lambda\right) \sim \frac{4 \pi}{\log |\Lambda|} \quad \text { as } \Lambda \rightarrow \mathbb{Z}^{2} .
$$

Indeed, the right-hand side is the probability that a particle detaching itself from the critical droplet reaches $\partial \Lambda$ and exits $\Lambda$ before reattaching itself. This probability is asymptotically independent of the shape and the location of the critical droplet, due to the fact that the free particle moves like a twodimensional simple random walk (which is recurrent on $\mathbb{Z}^{2}$ ). By reversibility, the reverse motion has the same probability, which is the left-hand side. Combining (39-41), we get (36).

In Theorem 2.1(a), an example of a configuration in $\mathcal{S} \backslash \mathcal{C}^{*}$ is an $\ell_{c} \times\left(\ell_{c}-1\right)$ quasi-square plus a dimer at distance 1 . For the dynamics, this configuration is a dead-end. Indeed, one particle of the dimer must jump back to the droplet and create a protuberance (at cost 0 ), and the remaining free particle must attach itself next to this protuberance (at gain $U$ ) to initiate the motion downhill to the $\ell_{c} \times \ell_{c}$ square. 


\subsection{Potential-theoretic approach}

We give a sketch of the techniques that are used to obtain the fine asymptotics of the average crossover time in Theorems $2.1(\mathrm{~d}-\mathrm{e})$ and $2.2(\mathrm{~d}-\mathrm{e})$. A key role is played by the notion of capacity between two sets of configurations, in particular, between the metastable state and the stable state. We refer to Section 3-5 in Bovier [4] for the general background of this notion within the context of metastability and for other applications.

Define

$$
\mathcal{E}(h)=\frac{1}{2} \sum_{\eta, \eta^{\prime} \in \mathcal{X}} \mu(\eta) c\left(\eta, \eta^{\prime}\right)\left[h(\eta)-h\left(\eta^{\prime}\right)\right]^{2}, \quad h: \mathcal{X} \rightarrow[0,1] .
$$

This is the Dirichlet form associated with the dynamics, whose argument is a potential function on the configuration space $\mathcal{X}$. Given two non-empty disjoint sets $\mathcal{A}, \mathcal{B} \subset \mathcal{X}$, the capacity of the pair $\mathcal{A}, \mathcal{B}$ is defined as

$$
\operatorname{CAP}(\mathcal{A}, \mathcal{B})=\min _{\substack{h:\left.\mathcal{X} \rightarrow[0,1] \\ h\right|_{\mathcal{A}} \equiv 1,\left.h\right|_{\mathcal{B}} \equiv 0}} \mathcal{E}(h),
$$

where the infimum runs over all potential functions whose restriction to $\mathcal{A}$ and $\mathcal{B}$ equals 1 and 0 , respectively. If we think of an electric network with nodes labelled by $\mathcal{X}$ and with conductivities $\mu(\eta) c\left(\eta, \eta^{\prime}\right)$ between nodes $\eta, \eta^{\prime} \in \mathcal{X}$, then $\mathcal{E}(h)$ is the energy produced by an electric current flowing through this network when the potential on the nodes is given by $h$. The capacity is the minimal energy when the nodes of $\mathcal{A}$ are kept at potential 1 and the nodes of $\mathcal{B}$ are kept at potential 0 ("Thompson's principle"). The minimum in (43) is unique, and the minimizer $h^{*}$ has the interpretation

$$
h^{*}(\eta)=\mathbb{P}_{\eta}\left(\tau_{\mathcal{A}}<\tau_{\mathcal{B}}\right) \quad \text { for } \eta \notin A \cup B .
$$

What is important about (43) is that upper bounds can be obtained by inserting test functions for $h$, while lower bounds can be obtained by removing transitions from $\mathcal{X} \times \mathcal{X}$ ("Rayleigh's short-cut rule"). This gives great flexibility in the calculations.

We henceforth focus on model (II), but the claims made below apply equally well to model (I). A key ingredient is the following fact, implying that $\{\square, \boldsymbol{\square}\}$ is a metastable pair for low temperature.

Proposition 2.1. (den Hollander, Nardi, Olivieri and Scoppola [18])

For all $\eta \in \mathcal{X} \backslash\{\square, \mathbf{\square}\}$,

$$
\Gamma(\eta,\{\square, \mathbf{\square}\})<\Gamma
$$

where

$$
\Gamma(\mathcal{A}, \mathcal{B})=\min _{\eta \in \mathcal{A}, \eta^{\prime} \in \mathcal{B}} \min _{\omega: \eta \rightarrow \eta^{\prime}} \max _{\xi \in \omega}[H(\xi)-H(\eta)]
$$

is the communication height between $\mathcal{A}, \mathcal{B} \subset \mathcal{X}, \mathcal{A} \cap \mathcal{B}=\emptyset, \mathcal{A}, \mathcal{B} \neq \emptyset$. 
Proposition 2.1 implies that no matter where the dynamics starts, it reaches the set $\{\square, \boldsymbol{\square}\}$ faster than it manages to achieve the crossover from $\square$ to 口. In words, there are "no deep pits" in the energy landscape that trap the dynamics for a time comparable to the crossover time.

The key to the fine estimate in Theorem $2.2(\mathrm{~d}-\mathrm{e})$ is the following fact, relating the average crossover time to the capacity and relying crucially on Proposition 2.1.

Proposition 2.2. (Bovier, den Hollander and Nardi [8]) $\mathbb{E}_{\square}(\tau \boldsymbol{\square})=[1+o(1)] /[Z \operatorname{CAP}(\square, \boldsymbol{\square})]$ as $\beta \rightarrow \infty$.

Thus, to estimate the average crossover time from $\square$ to $\mathbf{\square}$, it suffices to estimate the capacity of the pair $\square, \boldsymbol{\square}$. This proceeds in several steps.

(1) A crude a priori estimate yields that for every pair $\mathcal{A}, \mathcal{B}$ there exist constants $0<C_{1}<C_{2}<\infty$ (depending on $\mathcal{A}, \mathcal{B}$ but not on $\beta$ ) such that

$$
C_{1} \leq e^{\beta \Gamma(\mathcal{A}, \mathcal{B})} Z \operatorname{CAP}(\mathcal{A}, \mathcal{B}) \leq C_{2} .
$$

The lower bound is obtained by picking a minimax path $\omega$ in (46) and from (42) remove all transitions $\eta \rightarrow \eta^{\prime}$ that are not in $\omega$. The upper bound is obtained by picking a test function $h$ in (43) that is $\equiv 1$ on the $\Gamma$-valley around $\mathcal{A}$ and $\equiv 0$ on the $\Gamma$-valley around $\mathcal{B}$. (The $\Gamma$-valley around a set of configurations $\mathcal{S}$ is the set of configurations $\mathcal{S}^{\prime} \supsetneq \mathcal{S}$ whose communication height with $\mathcal{S}$ is $<\Gamma$.)

(2) With the help of (47), it is possible to obtain sharp bounds on the minimizer $h^{*}$ of (43) via so-called renewal-type estimates. These estimates show that $h^{*}$ is exponentially close (in $\beta$ ) to 1 on the $\Gamma$-valley around $\square$ and exponentially close (in $\beta$ ) to 0 on the $\Gamma$-valley around $\boldsymbol{\square}$. Since the configurations with energy $>\Gamma$ are negligible, because of the Gibbs factor in (42), it follows that the sharp asymptotics of $\operatorname{CAP}(\square, \boldsymbol{\square})=\mathcal{E}\left(h^{*}\right)$ is determined by the values of $h^{*}$ on $\mathcal{S}=\mathcal{S}(\square, \boldsymbol{\square})$ and on $\partial^{e x t} \mathcal{S}$, the exterior boundary of $\mathcal{S}$.

(3) Due to the above, the variational problem in (43) on the full configuration space $\mathcal{X}$ reduces to a variational problem restricted to $\mathcal{S} \cup \partial^{e x t} \mathcal{S}$. This reduced variational problem has a much simpler structure, and can be understood in terms of the geometry of the configurations that are critical droplets or are close to critical droplets.

(4) For Kawasaki, the reduced variational problem involves the creation of a free particle when the droplet is protocritical, the motion of this free particle towards the droplet, and the attachment itself. Since this is a problem about simple random walk travelling between $\partial \Lambda$ and a protocritical droplet somewhere inside $\Lambda$, the reduced capacity can be sharply estimated.

For Kawasaki, $\mathcal{S} \cup \partial^{e x t} \mathcal{S}$ contains plateaus, wells and dead-ends, and hence a closed form computation of $K=K\left(\ell_{c}, \Lambda\right)$ is not feasible. Fortunately, for 
large $\Lambda$ the details of the geometry of $\mathcal{S} \cup \partial^{\text {ext }} \mathcal{S}$ turn out to be only partly relevant, and the asymptotics of $K$ can be identified, resulting in (36). For Glauber, the reduced variational problem turns out to be zero-dimensional, and $K=K\left(\ell_{c}, \Lambda\right)$ can be computed in closed form, resulting in (25).

Remark: Most of the results desribed above can be extended to other types of dynamics, such as Glauber dynamics for Ising spins with an anisotropic interaction or in a staggered magnetic field, or Ising spins subject to a parallel dynamics given by a probabilistic cellular automaton (see den Hollander [17] for references). Similarly, most of the results can be extended to three dimensions, despite the more complex geometry of critical droplets (see Ben Arous and Cerf [2], den Hollander, Nardi, Olivieri and Scoppola [18] for the necessary background).

\section{Lecture 3: Large systems at low temperature and moderate systems at positive temperature, conjectures}

In this lecture we move away from finite systems and investigate what happens in growing volumes, both at low and at positive temperature. Most of what is described below consists of target theorems and work in progress.

Glauber dynamics for large systems at low temperature was studied in Dehghanpour and Schonmann [12], [13], Schonmann and Shlosman [30], and Manzo and Olivieri [21], using the pathwise approach. Current work focusses on trying to improve their results using the potential-theoretic approach, and on extending the analysis to Kawasaki dynamics.

\subsection{Large systems at low temperature}

\section{Glauber dynamics}

Let $\Lambda=\Lambda_{\beta}$ depend on $\beta$ such that

$$
\left|\Lambda_{\beta}\right|=e^{\Theta \beta}, \quad \Theta \in[0, \infty) .
$$

Let

- $\mathcal{R} \subset \mathcal{X}$ denote those configurations where the circumscribed rectangles of all clusters of up-spins in $\Lambda_{\beta}$ are contained in non-interacting protocritical quasi-squares (recall Fig. 9).

The initial configuration $X(0)$ of the dynamics is drawn according to the conditional Gibbs measure

$$
\mu_{\mathcal{R}}(\eta)=\frac{\mu(\eta) 1_{\mathcal{R}}(\eta)}{\mu(\mathcal{R})}, \quad \eta \in \mathcal{X}_{\beta},
$$




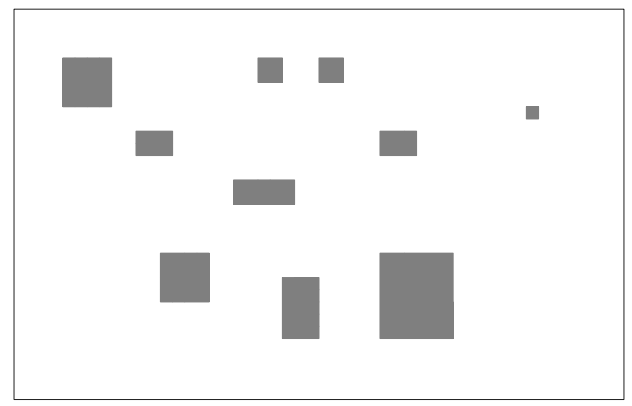

Fig. 9. A configuration in $\mathcal{R}$.

where $\mu$ is defined in (7) and $\mu(\mathcal{R})=\sum_{\eta \in \mathcal{R}} \mu(\eta)$. Our goal will be to estimate the first time a critical droplet appears anywhere in $\Lambda_{\beta}$, i.e., the first exit time of $\mathcal{R}$.

As before, we will be interested in the metastable regime

$$
h \in(0,2 J), \quad \beta \rightarrow \infty .
$$

Write $\mathbb{P}_{\eta}$ to denote the law of the dynamics $X=(X(t))_{t \geq 0}$ starting from $X(0)=\eta$, and put $\mathbb{P}_{\mu_{\mathcal{R}}}=\sum_{\eta \in \mathcal{R}} \mu_{\mathcal{R}}(\eta) \mathbb{P}_{\eta}$. Let

$$
\tau_{\mathcal{R}^{c}}=\min \{t \geq 0: X(t) \notin \mathcal{R}\}
$$

denote the first time the dynamics exits $\mathcal{R}$. Write $\asymp$ for asymptotic equality modulo constants.

Conjecture 3.1. (Bovier, den Hollander and Spitoni [9])

If

$$
\Theta \in[0, \Gamma-\Xi) \quad \text { with } \quad \Xi=h\left(\ell_{c}-2\right),
$$

then

$$
\mathbb{E}_{\mu_{\mathcal{R}}}\left(\tau_{\mathcal{R}^{c}}\right) \asymp \frac{1}{\left|\Lambda_{\beta}\right|} e^{\beta \Gamma} \quad \text { as } \beta \rightarrow \infty .
$$

The idea behind Conjecture 3.1 is simple. The dynamics grows and shrinks droplets essentially independently in different local boxes. Consequently, a critical droplet appears randomly in one of the local boxes, after a time that is the local crossover time divided by the number of local boxes in $\left|\Lambda_{\beta}\right|$. This is the regime of homogeneous nucleation.

$\Gamma$ is the local energy of the critical droplet, which plays the role of the local activation energy for the crossover. $\Xi$ is the local energy needed to evaporate the largest subcritical droplet. The regime in (52) corresponds to the situation where any subcritical droplet has a tendency to evaporate in a time much smaller than the crossover time. 


\section{Kawasaki dynamics}

Keep (48). This time, let

- $\mathcal{R} \subset \mathcal{X}$ denote those configurations where all clusters of particles in $\Lambda_{\beta}$ are either strictly contained in a protocritical quasi-square plus protuberance (recall Fig. 7), or are equal to a protocritical quasi-square plus protuberance with an empty annulus of size, say, $10 \ell_{c}$ (see Fig. 10).

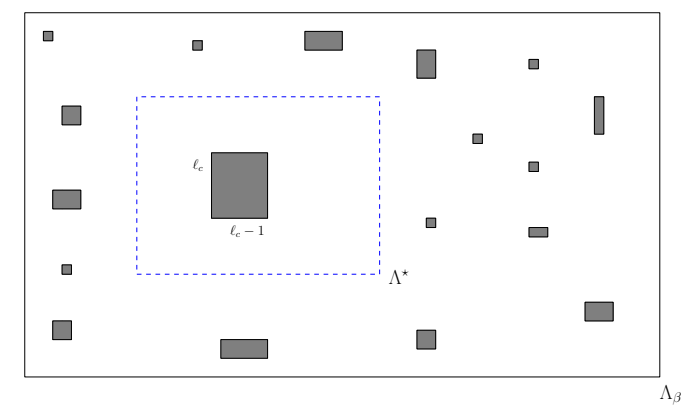

Fig. 10. A configuration in $\mathcal{R}$.

The initial configuration $X(0)$ of the dynamics is again drawn according to (49). We run the dynamics associated with the Hamiltonian in the second line of (1) without the activity term. Indeed, this term is no longer needed, because $\Lambda_{\beta}$ is so large that it takes over the role of the gas reservoir. In fact, we will supply $\Lambda_{\beta}$ with periodic boundary conditions, so that no particle enters or exits $\Lambda_{\beta}$ at positive times. Our choice to start from the conditional equilibrium with activity, given by (49), is needed at time zero only, and is made for convenience. Thus, the particle density inside $\Lambda_{\beta}$ is $e^{-\beta \Delta}$ at time zero and remains fixed in the course of time.

In order to have particles at all we must pick $\Theta>\Delta$. We will be interested in the regime

$$
\Delta \in(U, 2 U), \quad \Theta \in(\Delta, \infty), \quad \beta \rightarrow \infty .
$$

Conjecture 3.2. (Gaudilliere, den Hollander, Nardi, Olivieri and Scoppola [16], Bovier, den Hollander and Spitoni [9])

Suppose that $\ell_{c} \geq 3$. If

$$
\Theta \in(\Delta, \Gamma-\Xi) \quad \text { with } \quad \Xi=2 U+\left(\ell_{c}-3\right)(2 U-\Delta)
$$

then

$$
\mathbb{E}_{\mu_{\mathcal{R}}}\left(\tau_{\mathcal{R}^{c}}\right) \asymp \beta \frac{1}{\left|\Lambda_{\beta}\right|} e^{\beta \Gamma} \quad \text { as } \beta \rightarrow \infty .
$$

Because of the low particle density, as before, droplets grow and shrink more or less independently in different local boxes, causing homogeneous nucleation. 


\subsection{Moderate systems at positive temperature}

In equilibrium statistical physics, for a system that is at a first-order phase transition a macroscopically large droplet of one phase inside the other phase takes on the Wulff shape, i.e., the droplet minimizes its total surface tension subject to a total volume constraint. This observation, which is over a century old, has been put on a rigorous microscopic basis since only fifteen years or so. For the two-dimensional ferromagnetic nearest-neighbor Ising model at low temperature, Dobrushin, Kotecký and Shlosman [14] proved that a large droplet of the plus-phase inside the minus-phase has the Wulff shape. This result was subsequently extended up to the critical temperature, and its proof was simplified, by Pfister [26], Ioffe [20] and Pisztora [27].

The Wulff construction requires a careful coarse-graining analysis. The microscopic phase boundary is approximated on a mesoscopic scale by a polygon consisting of many segments, which decouple on the mesoscopic scale. Each segment contributes to the surface tension in a way that depends on its direction relative to the lattice axes. To handle the fluctuations of the boundary around the polygon, large deviation arguments are required. The polygon tends to a smooth curve in the macroscopic limit, and this curve enters into the Wulff variational problem, whose solution is the actual phase boundary.

To study Wulff droplets in the presence of a stochastic dynamics is part of non-equilibrium statistical physics and therefore is quite a different matter. The question of interest is whether macroscopically large critical droplets for metastable transitions between two phases under a stochastic local dynamics assume the Wulff shape or not.

In this lecture we allow the box $\Lambda$ to grow but only moderately, in a way that depends not on $\beta$ but on the parameters in the Hamiltonian.

\section{Glauber dynamics}

We suppose that $\Lambda=\Lambda_{h}$ with

$$
\left|\Lambda_{h}\right|=C \frac{1}{h}, \quad 1 \ll C<\infty
$$

We assume that $\beta>\beta_{c}$, the critical inverse temperature at $h=0$ for $\Lambda=\mathbb{Z}^{2}$. The system starts at $X(0)=\boxminus$, the Glauber dynamics is applied for small $h>0$, and the limit $h \downarrow 0$ is taken. The dynamics eventually brings the system to equilibrium, close to the plus-phase at $h=0$, but it needs a long time to do so. In the limit $h \downarrow 0$, the critical droplet becomes macroscopically large. The goal is to show that the critical droplet scales to the equilibrium Wulff shape and appears after a time that scales like the exponential of the Wulff

free energy. The size of the box is taken to scale in such a way that the critical droplet occupies a finite fraction of the box. 
Theorem 3.1. (Schonmann and Shlosman [30])

For $\beta>\beta_{c}$ and $C$ sufficiently large,

$$
\lim _{h \downarrow 0} h \log \mathbb{E}_{\boxminus}\left(\tau_{m(J, \beta)}\right)=\frac{W(J, \beta)^{2}}{4 m(J, \beta)},
$$

where $\tau_{m(J, \beta)}$ is the first time the total magnetization inside $\Lambda_{h}$ equals $m(J, \beta)$, the spontaneous magnetization on $\mathbb{Z}^{2}$, and $W(J, \beta)$ is the total surface tension of the Wulff droplet of unit volume.

Note that the left-hand side of (58) refers to a non-equilibrium quantity, while the right-hand side only contains quantities from equilibrium. This is why the result in (58) is deep.

The idea behind (58) is that, in the macroscopic scaling limit, the critical droplet has a length $\ell$ that maximizes the free energy function

$$
f(\ell)=-m(J, \beta) h \ell^{2}+W(J, \beta) \ell .
$$

This is a macroscopic version of the parabola encountered in (15)!) The maximum is taken at $\ell_{\max }=W(J, \beta) / 2 m(J, \beta) h$, giving free energy

$$
f\left(\ell_{\max }\right)=\frac{W(J, \beta)^{2}}{4 m(J, \beta) h} .
$$

This is the exponential of the time needed to create a droplet at a given location.

Schonmann and Shlosman [30] analyze the problem also on $\mathbb{Z}^{2}$ instead of on $\Lambda_{h}$ subject to (57). They show that, in infinite volume, the critical droplet typically is not created close to the origin, but rather is created far away and subsequently invades the origin by growing. As a result, the exponential is three times smaller, because the critical droplet may occur anywhere in a space-time cone of this smaller size and invade the origin afterwards.

\section{Kawasaki dynamics}

This time we suppose that $\Lambda=\Lambda_{\Delta}$ with

$$
\left|\Lambda_{\Delta}\right|=C \frac{1}{2 U-\Delta}, \quad 1 \ll C<\infty .
$$

We assume that $\beta>\beta_{c}$, the critical inverse temperature for the Hamiltonian without activity term for $\Lambda=\mathbb{Z}^{2}$. The system starts at $X(0)=\square$, the Kawasaki dynamics is applied for $\Delta<2 U$, and the limit $\Delta \uparrow 2 U$ is taken. This is the limit of weak supersaturation, when the critical droplet becomes macroscopically large. 
Conjecture 3.3. (Bovier, den Hollander and Ioffe [7])

For $\beta>\beta_{c}$ and $C$ sufficiently large,

$$
\lim _{\Delta \uparrow 2 U}(2 U-\Delta) \log \mathbb{E}_{\square}\left(\tau_{\rho(U, \beta)}\right)=\frac{W(U, \beta)^{2}}{2 \rho(\beta, U)-1},
$$

where $\tau_{\rho(U, \beta)}$ is the first time the particle density inside $\Lambda_{\Delta}$ equals $\rho(U, \beta)$, the density of the liquid phase on $\mathbb{Z}^{2}$, and $W(U, \beta)$ is the total surface tension of the Wulff droplet of unit volume.

The right-hand side of (62) is the same as that of (60), with $J$ being replaced by $U / 2$, because of the link between the Hamiltonians of models (I) and (II) in (1). The reason is that, as already observed above, the right-hand side of (62) only contains quantities from equilibrium.

A proof of Conjecture 3.3 is currently being attempted, with the help of the potential-theoretic techniques mentioned in Section 2.3, for a version of the model where the interaction is of Kac-Dyson type, i.e., quasi-mean-field. The hard part is that, for growing volumes at positive temperature, both spatial and temporal entropy need to be controlled. We need to understand the typical way in which the dynamics grows and shrinks large droplets, absorbing and emitting large numbers of particles with the surrounding gas phase in the box while keeping the droplet close to the Wulff shape. Droplets are expected to grow and shrink via "motion by curvature".

\section{References}

1. L. Alonso and R. Cerf, The three-dimensional polyominoes of minimal area, Electron. J. Combin. 3 (1996) Research Paper 27.

2. G. Ben Arous and R. Cerf, Metastability of the three-dimensional Ising model on a torus at very low temperature, Electron. J. Probab. 1 (1996) Research Paper 10 .

3. A. Bovier, Metastability: a potential theoretic approach, Proceedings of ICM 2006, Vol. 3, EMS Publising House, 2006, pp. 499-518.

4. A. Bovier, Metastability. In this volume. Springer, Berlin, 2007.

5. A. Bovier, M. Eckhoff, V. Gayrard and M. Klein, Metastability in stochastic dynamics of disordered mean-field models, Probab. Theory Rel. Fields 119 (2001) 99-161.

6. A. Bovier, M. Eckhoff, V. Gayrard and M. Klein, Metastability and low lying spectra in reversible Markov chains, Commun. Math. Phys. 228 (2002) 219-255.

7. A. Bovier, F. den Hollander and D. Ioffe, work in progress.

8. A. Bovier, F. den Hollander and F.R. Nardi, Sharp asymptotics for Kawasaki dynamics on a finite box with open boundary, Probab. Theory Relat. Fields 135 (2006) 265-310.

9. A. Bovier, F. den Hollander and C. Spitoni, work in progress.

10. A. Bovier and F. Manzo, Metastability in Glauber dynamics in the lowtemperature limit: beyond exponential asymptotics, J. Stat. Phys. 107 (2002) $757-779$. 
11. M. Cassandro, A. Galves, E. Olivieri and M.E. Vares, Metastable behavior of stochastic dynamics: a pathwise approach, J. Stat. Phys. 35 (1984) 603-634.

12. P. Dehghanpour and R.H. Schonmann, Metropolis dynamics relaxation via nucleation and growth, Comm. Math. Phys. 188 (1997) 89-119.

13. P. Dehghanpour and R.H. Schonmann, A nucleation-and-growth model, Probab. Theory Rel. Fields 107 (1997) 123-135.

14. R.L. Dobrushin, R. Kotecký, S.B. Shlosman, Wulff construction: a global shape from local interaction, AMS translations series, Providence, RI, Am. Math. Soc. 1992.

15. M.I. Freidlin and A.D. Wentzell, Random Perturbations of Dynamical Systems, Springer, Berlin, 1984.

16. A. Gaudillière, F. den Hollander, F.R. Nardi, E. Olivieri and E. Scoppola, work in progress.

17. F. den Hollander, Metastability under stochastic dynamics, Stoch. Proc. Appl. 114 (2004) 1-26.

18. F. den Hollander, F.R. Nardi, E. Olivieri and E. Scoppola, Droplet growth for three-dimensional Kawasaki dynamics, Probab. Theory Relat. Fields 125 (2003) 153-194.

19. F. den Hollander, E. Olivieri and E. Scoppola, Metastability and nucleation for conservative dynamics, J. Math. Phys. 41 (2000) 1424-1498.

20. D. Ioffe, Exact deviation bounds up to $T_{c}$ for the Ising model in two dimensions, Probab. Theory Relat. Fields 102 (1995) 313-330.

21. F. Manzo and E. Olivieri, Dynamical Blume-Capel model: competing metastable states at infinite volume, J. Stat. Phys. 104 (2001) 1029-1090.

22. E.J. Neves and R.H. Schonmann, Critical droplets and metastability for Glauber dynamics at very low temperature, Comm. Math. Phys. 137 (1991) 209-230.

23. E. Olivieri and E. Scoppola, Metastability and typical exit paths in stochastic dynamics, Proceedings of ECM 1996, Progress in Mathematics 169, Birkhäuser, Basel, 1998, pp. 124-150.

24. E. Olivieri and M.E. Vares, Large Deviations and Metastability, Cambridge University Press, Cambridge, 2004.

25. O. Penrose and J.L. Lebowitz, Towards a rigorous molecular theory of metastability, in: Fluctuation Phenomena, 2nd. ed. (E.W. Montroll and J.L. Lebowitz, eds.), North-Holland, Amsterdam, 1987.

26. C.E. Pfister, Large deviations and phase separation in the two-dimensional Ising model, Helv. Phys. Acta 64 (1991) 953-1054.

27. A. Pisztora, Surface order large deviations for Ising, Potts and percolation models, Probab. Theory Relat. Fields 104 (1996) 427-466.

28. R.H. Schonmann, Theorems and conjectures on the droplet-driven relaxation of stochastic Ising models, in: Probability and Phase Transitions (G. Grimmett ed.), NATO ASI Series, Kluwer Academic Publishers, Dordrecht, 1994, pp. 265301.

29. R.H. Schonmann, Metastability and the Ising model, Proceedings of ICM 1998, Doc. Math., Extra Vol. 3, pp. 173-181.

30. R.H. Schonmann, S.B. Shlosman, Wulff droplets and the metastable relaxation of kinetic Ising models, Comm. Math. Phys. 194 (1998) 389-462.

31. E. Scoppola, Metastability for Markov chains, in: Probability and Phase Transitions (G. Grimmett ed.), NATO ASI Series, Kluwer Academic Publishers, Dordrecht, 1994, pp. 303-322. 
32. M.E. Vares, Large deviations and metastability, in: Disordered Systems, Traveaux en Cours 53, Hermann, Paris, 1996, pp. 1-62. 